\title{
Dendrite-Hardened Amorphous and Graphene-Reinforced Metal Composites: Deformation Mechanisms and Strength Characteristics
}

\author{
V. A. Polukhin ${ }^{1,2, a)}$, S. Kh. Estemirova ${ }^{1,2}$, and E. D. Kurbanova ${ }^{1}$ \\ ${ }^{1}$ Institute of Metallurgy, Ural Branch of the Russian Academy of Sciences, 101 Amundsena St., Ekaterinburg, \\ 620016, Russia \\ ${ }^{2}$ Ural Federal University, 19 Mira St., Ekaterinburg, 620002, Russia \\ ${ }^{a)}$ Corresponding author: p.valery47@yandex.ru
}

\begin{abstract}
The deformation mechanisms of hardened amorphous alloys and graphene-reinforced nanolayer composites are studied by molecular dynamics modeling (MD). It has been established (using $\mathrm{CuZrAl}$ as an example) that in amorphous alloys, under the influence of intense deformations accompanied by heat release, a dendritic nanophase is formed, which contributes to their strengthening. The dendritic nanophase hardens the alloys by tensile strength up to $20 \%$ at loads of $2300 \mathrm{MPa}$; the yield strength exceeds $1550 \mathrm{MPa}$. In graphene-reinforced nanolayer composites $(\mathrm{Me} /$ graphene, where $\mathrm{Me}=\mathrm{Ni}, \mathrm{Al}, \mathrm{Ti})$, a deformation mechanism of the transfer type is realized. The transfer-type mechanism is the appearance of uniformly distributed microscopic cracks ("strain transfer") on the surface of the sample (along its entire length). In composites subjected to such deformation, the strength characteristics are significantly increased. The simulation results show good agreement (10 to $15 \%$ ) with experimental literature data.
\end{abstract}

\section{INTRODUCTION}

Currently, amorphous alloys (metallic glasses) and metal/graphene (Me/G) nanolayer composites are used not only in nano- and spintronics, but also as structural materials in reactors due to their resistance to radiation damage [4-6]. In metallic glasses with predominantly metallic interatomic bonds and the absence of translational symmetry, deformation loads lead to stress dissipation with the rearrangement of structural cluster inhomogeneities (classified as $\tau$-defects [6-8]). Similar processes under the action of deformation loads occur in graphene-reinforced nanocomposites [8]. In the process of smooth relaxation of stresses at the atomic level, the short-range (topological) order is reformed and the local free volumes are redistributed. In fact, stress-induced relaxation occurs in atomic cluster groups with a smooth shear configurational transformation and energy dissipation into the local environment. Zones of deformation shear are represented by planar layers of atomic rows, shifted from their original locations when a thermal or deforming front passes (Fig. 1).

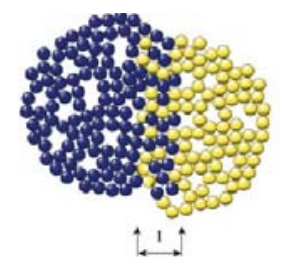

(a)

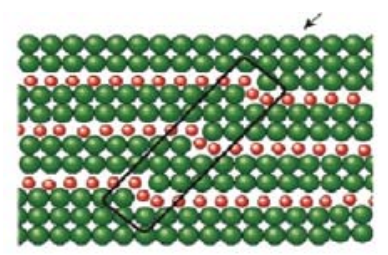

(b)

FIGURE 1. Schematic representation of the relaxation of STZ zonal shear loads of transformation and compaction for dendritestrengthened amorphous (a) and graphene-reinforced (b) metal composites: the arrows indicate the bands of zonal shears caused by the loads [7] 
In the present work we have carried out a theoretical MD simulation study of the strength and thermal stability of metallic glasses and nanocomposites reinforced by layered graphene. The results are compared with experimental data.

\section{RESULTS AND DISCUSSION}

Under the influence of deformations, diffusion transport of atoms, the movement of point and linear defects, vortex disclinations, and gliding dislocations are activated. Each of these mechanisms depends on the composition of the samples, the method of their preparation, morphology, degree of crystallinity, microstructure parameters (the shape and size of grains, the presence of a dendritic nanophase, the state of grain-boundary surfaces), and the nature of stacking of graphene-reinforced layered compositions. In addition, the intensity (speed) of the applied deforming load plays a significant role. It was established by the method of MD simulation that the thermodynamic state of the samples subjected to deforming load passes through a number of metastable states, following, as it were, in the likeness of a potential "landscape", Fig. 2 [6,7]. Thus, the strength characteristics (stress $\sigma$ and elongation $\varepsilon \sim \Delta 1 / 1)$ of the simulated amorphous $\mathrm{Zr}-\mathrm{Cu}$-Ti films obtained from the initial melts improved with an increase in the cooling rate (up to $100 \mathrm{~K} / \mathrm{s}$ ). In this case, the thermal stability of the amorphous phases was retained [1-3, 10]. The reinforcement of amorphous alloys based on $\mathrm{ZrCuTi}$ with graphene layers significantly changes the nature of deformations (Fig. 2). Arrow 1 in Fig. 2 shows the deformation-induced change for the $\mathrm{Zr}_{41.2} \mathrm{Ti}_{13.8} \mathrm{Cu}_{12.5} \mathrm{Ni}_{10} \mathrm{Be}_{22}$ glass) $[6,8]$, and arrow 2 (our MD simulation results) shows deformation under the same loads applied to simulated samples of amorphous alloys of the same composition, reinforced with graphene layers.

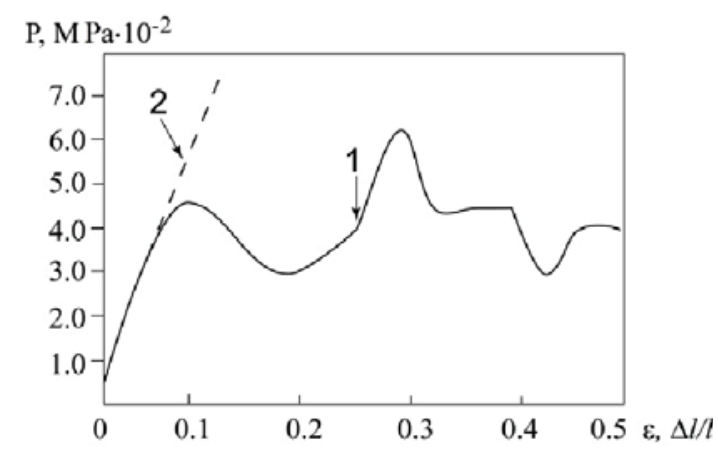

FIGURE 2. The dependence of deformation $\varepsilon$ on the applied load $\mathrm{P} \sim 4.5 \cdot 10^{-2} \mathrm{MPa}$ with a rate $\mathrm{d} \varepsilon / \mathrm{dt} \sim 10^{-2} / \mathrm{s}$ (at a temperature $\mathrm{T} \sim 650 \mathrm{~K} ; \mathrm{T} / \mathrm{Tg}=1.03$ ); arrows 1 and 2 - explanation in the text

As a result of deformation and the associated thermal effect, partial devitrification of the amorphous phase occurs with the formation of strengthening dendritic nanophases formed from coherently joined fragments of spiral and high-density icosahedral configurations, Fig. 3.

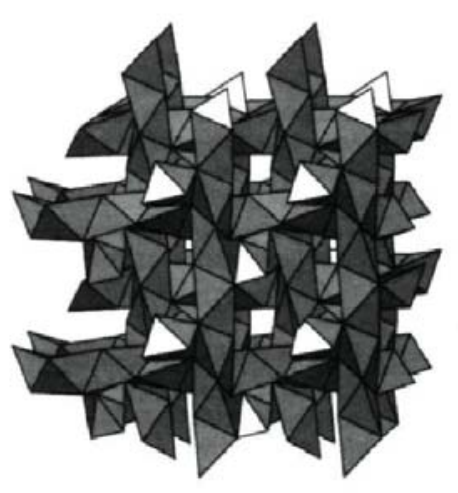

(a)

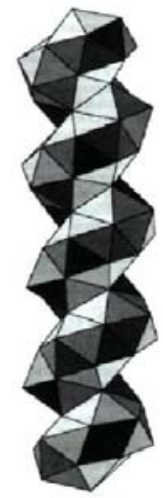

(b)

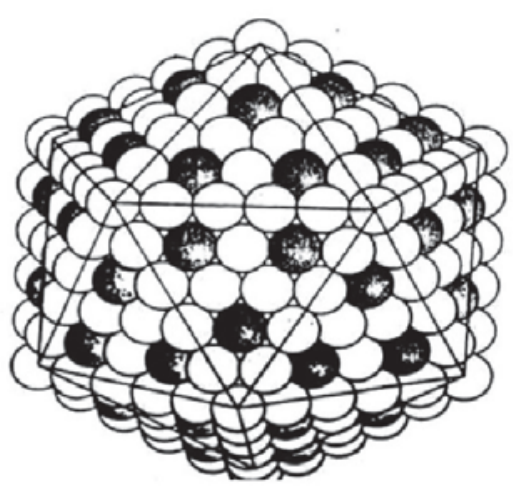

(c)

FIGURE 3. Constructions of rod nanodendritic spiral substructures strengthened the amorphous matrix, composed of regular tetrahedra (a), helicoids (b), and close-packed icosahedra (c) 
We have made calculations within the molecular dynamics simulation in accordance with the procedure described in [9] for metal/graphene interface pairs $(\mathrm{G} / \mathrm{Me}, \mathrm{Me}=\mathrm{Ni}, \mathrm{Cu}, \mathrm{Ti}, \mathrm{Ru})$, which resulted in high values of cohesive energy $\left(E_{C}\right)$. For example, $E_{C}=-91.33 \cdot 10^{2} \mathrm{eV} \mathrm{nm}^{-2} / \mathrm{C}$ for the $\mathrm{G} / \mathrm{Ni}$ contact, and $\mathrm{E}_{\mathrm{C}}=-24.81 \cdot 10^{2} \mathrm{eV} \mathrm{nm}^{-}$ ${ }^{2} / \mathrm{C}$ for the $\mathrm{G} / \mathrm{Cu}$ contact. The tensile strength is $2.92 \mathrm{GPa}$ for $\mathrm{Cu} / \mathrm{G}$ and $18.70 \mathrm{GPa}$ for $\mathrm{Ni} / \mathrm{G}$.
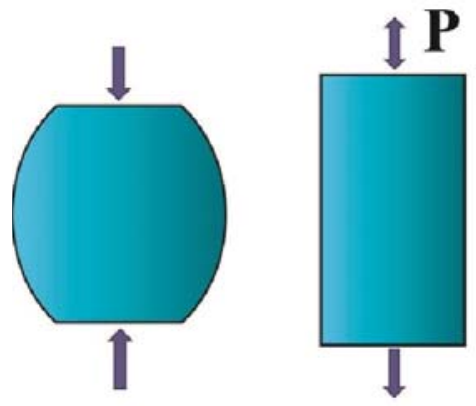

(a)
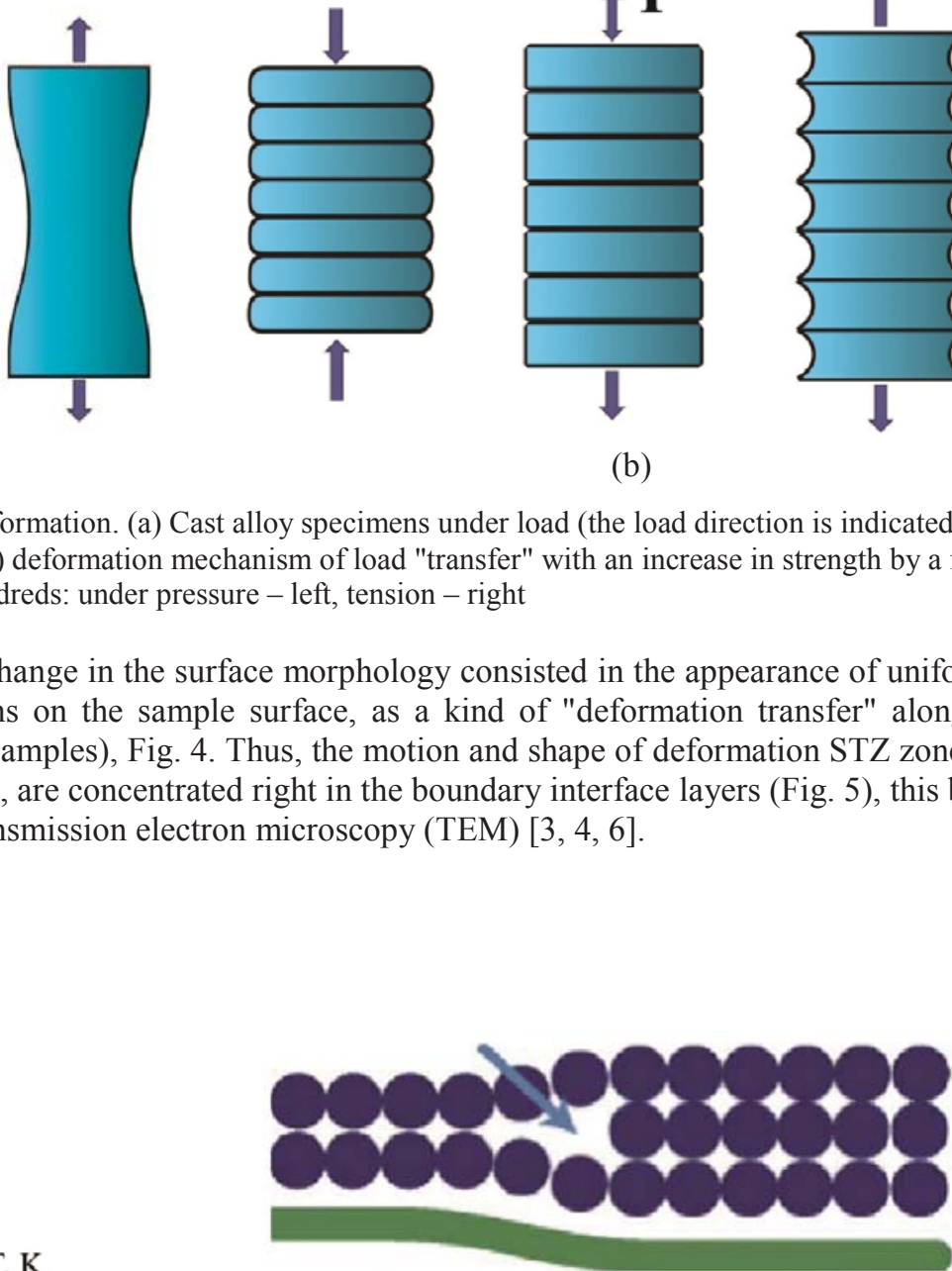

(b)

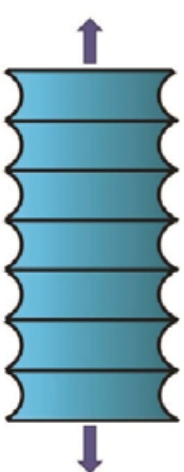

(b)

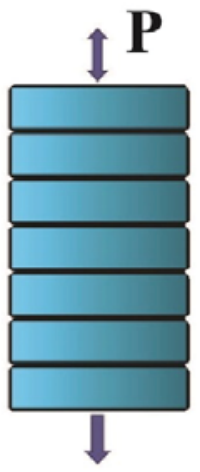

FIGURE 4. Schematic representation of deformation. (a) Cast alloy specimens under load (the load direction is indicated by arrows): compression - left, tension - right; (b) deformation mechanism of load "transfer" with an increase in strength by a factor of hundreds: under pressure - left, tension - right
As a result of the loads, a qualitative change in the surface morphology consisted in the appearance of uniformly
force lines (along the entire length of the samples), Fig. 4. Thus, the motion and shape of deformation STZ zones, as
well as dislocation clusters of high density, are concentrated right in the boundary interface layers (Fig. 5), this being
consistent with observations by in-situ transmission electron microscopy (TEM) $[3,4,6]$. of hundreds: under pressure - left, tension - right
As a result of the loads, a qualitative change in the surface morphology consisted in the appearance of uniformly
distributed microscopic cracks-depressions on the sample surface, as a kind of "deformation transfer" along the
force lines (along the entire length of the samples), Fig. 4. Thus, the motion and shape of deformation STZ zones, as
well as dislocation clusters of high density, are concentrated right in the boundary interface layers (Fig. 5), this being
consistent with observations by in-situ transmission electron microscopy (TEM) [3, 4,6]. of hundreds: under pressure - left, tension - right
As a result of the loads, a qualitative change in the surface morphology consisted in the appearance of uniformly
distributed microscopic cracks-depressions on the sample surface, as a kind of "deformation transfer" along the
force lines (along the entire length of the samples), Fig. 4. Thus, the motion and shape of deformation STZ zones, as
well as dislocation clusters of high density, are concentrated right in the boundary interface layers (Fig. 5), this being
consistent with observations by in-situ transmission electron microscopy (TEM) [3, 4,6]. of hundreds: under pressure - left, tension - right
As a result of the loads, a qualitative change in the surface morphology consisted in the appearance of uniformly
distributed microscopic cracks-depressions on the sample surface, as a kind of "deformation transfer" along the
force lines (along the entire length of the samples), Fig. 4. Thus, the motion and shape of deformation STZ zones, as
well as dislocation clusters of high density, are concentrated right in the boundary interface layers (Fig. 5), this being
consistent with observations by in-situ transmission electron microscopy (TEM) [3, 4,6]. of hundreds: under pressure - left, tension - right
As a result of the loads, a qualitative change in the surface morphology consisted in the appearance of uniformly
distributed microscopic cracks-depressions on the sample surface, as a kind of "deformation transfer" along the
force lines (along the entire length of the samples), Fig. 4. Thus, the motion and shape of deformation STZ zones, as
well as dislocation clusters of high density, are concentrated right in the boundary interface layers (Fig. 5), this being
consistent with observations by in-situ transmission electron microscopy (TEM) [3, 4,6]. of hundreds: under pressure - left, tension - right
As a result of the loads, a qualitative change in the surface morphology consisted in the appearance of uniformly
distributed microscopic cracks-depressions on the sample surface, as a kind of "deformation transfer" along the
force lines (along the entire length of the samples), Fig. 4. Thus, the motion and shape of deformation STZ zones, as
well as dislocation clusters of high density, are concentrated right in the boundary interface layers (Fig. 5), this being
consistent with observations by in-situ transmission electron microscopy (TEM) [3, 4,6].

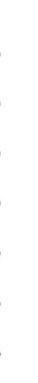

(a)

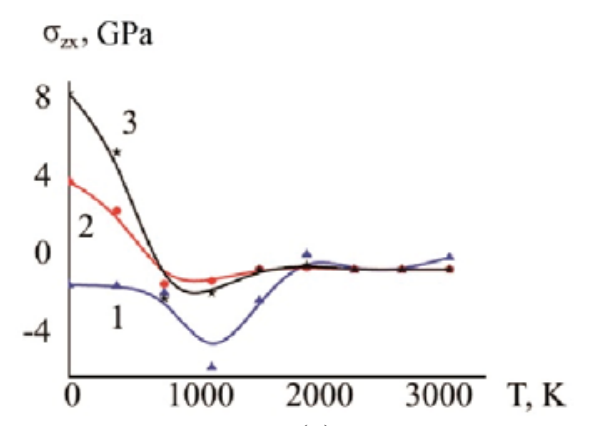

FIGURE 5. MD simulation results. (a) Temperature relaxation of stresses $\sigma_{\mathrm{zx}}$ in the plane of metal films: $1-\mathrm{Al}, 2-\mathrm{Ni}, 3-\mathrm{Ti}$;

(b) the mechanism of blocking the movements of dislocations (in layers) and shear deformation zones (in crystalline or amorphous nanofilms) by reinforcing graphene sheets

The interface contacts actually perform the function of effectively blocking creeping dislocation and disclination (sliding) motions. This impermeability of the G/Me interfaces is realized by the adjustment of graphene sheets with their deflection caused by the deformation load P (Fig. 5). This is followed by (a) relaxation with recoordination in metal layers adjacent to graphene and (b) with the formation of STZ bands in the graphene-reinforced composite [9, $10-12]$.

\section{CONCLUSION}

We have presented the results of simulated mechanical tests of amorphous multicomponent alloys based on $\mathrm{Cu}-$ $\mathrm{Zr}$, including high compression loads and significant plastic deformations with the appearance of faults in the samples. The specificity of the deformation mechanism consists in the formation of free volume in amorphous 
alloys, limitation of the development of the dynamics of point and linear defects; the formation of a defect structure is accompanied by heat release.

The high strength of the graphene-reinforced $\mathrm{Ti} / \mathrm{G}$ composite obtained as a result of MD simulation was achieved not only due to deformation transfer, but also due to very strongly hybridized Ti-C bonds in hexagonal planes (Young's modulus $\sim 1 \mathrm{TPa}$ ). The dislocations formed under the action of the deformation load in the graphene-reinforced $\mathrm{Ni} / \mathrm{G}$ composite are blocked and accumulated (i.e., they run off) in the second coordination plane of $\mathrm{Ni}$ with respect to graphene ("pinning"), without overcoming the "obstacles" of the C-C bond network, which is consistent with the TEM and STM data [3-5].

Thus, from the results of MD simulation, which are in good agreement with the experimental data, it follows that graphene-reinforced nanolayer composites, like dendritically-strengthened amorphous metal composites, do indeed have unique strength characteristics and high thermal stability.

\section{ACKNOWLEDGMENTS}

The work was performed according to the state assignment for IMET UB RAS, project No. 0396-2019-0002.

\section{REFERENCES}

1. V. A. Polukhin and N. A. Vatolin, Simulation of Disordered and Nanostructured Phases (UrO RAN Publ., Ekaterinburg, 2011).

2. R. Z. Valiev, A. P. Zhilyaev, and T. G. Langdon, Bulk Nanostructured Materials: Fundamentals and Applications (John Wiley and Sons, Inc.-TMS, Hoboken, NJ, 2013).

3. V. A. Polukhin, and N. A. Vatolin, Chem. Rev. 84(5), 498-539 (2015).

4. Y. Kim, J. Lee, M. S. Yeom, J. W. Shin, H. Kim, Y. Cui, J. W. Kysar, J. Hone, Y. Jung, S. Jeon, S. M. Yan, Nat. Commun. 4, 2114 (2013).

5. I. A. Ovid'ko, Reviews on Advanced Materials Science 38(2), 190-200 (2014).

6. R. T. Qu, Z. Q. Liu, G. Wang, and Z. F. Zhang, Acta Mater. 91, 19-33 (2015).

7. P. Thurnheer, F. Haag, and J. F. Loffer, Acta Mater. 115, 468-474 (2016).

8. V. A. Polukhin, N. I. Sidorov, and N. A. Vatolin, Russ. Metall. 2019(8), 758-780 (2019).

9. V. A. Polukhin, E. D. Kurbanova, and A. E. Galashev, "Proceedings of 14th International Conference on Liquid and Amorphous Metals-2010," EPJ Web of Conferences 15, 00001 (2011).

10. A. Inoue, A. Takeuchi, Acta Materialia 59(6), 2243-2267 (2011).

11.E. A. Pastukhov, N. I. Sidorov, V. A. Polukhin et al, "4th International Conference on Diffusion in Solids and Liquids-2008," Defect and Diffusion Forum 283-286, 149-154 (2009).

12. V. A. Polukhin and N. A. Vatolin, Russ. Metall. 2018(8), 685-699 (2018).

13. V. A. Polukhin, E. D. Kurbanova, and N. S. Mitrofanova, Russ. Metall. 2017(8), 116-126 (2017).

14. V. A. Polukhin, E. D. Kurbanova, and N. A. Vatolin, Russ. Metall. 2018(2), 95-109 (2018).

15. V. A. Polukhin and M. M. Dzugutov, Metal. Metallogr. 511, 50-55 (1981). 